\title{
NGAL: an emerging tool for predicting severity of AKI is easily detected by a clinical assay
}

\author{
Wes Gabbard,1 Eric B Milbrandt² and John A Kellum*3 \\ University of Pittsburgh Department of Critical Care Medicine: Evidence-Based Medicine Journal Club, edited by Eric B Milbrandt
}

\section{Expanded Abstract Citation}

Bennett M, Dent CL, Ma Q, Dastrala S, Grenier F, Workman R, Syed H, Ali S, Barasch J, Devarajan P: Urine NGAL predicts severity of acute kidney injury after cardiac surgery: a prospective study. Clin J Am Soc Nephrol 2008, 3:665-673 [1].

\section{Background}

The authors have previously shown that urine neutrophil gelatinase-associated lipocalin (NGAL), measured by a research ELISA, is an early predictive biomarker of acute kidney injury (AKI) after cardiopulmonary bypass (CPB). The availability of a standardized clinical platform for NGAL measurements could revolutionize renal diagnostics, especially in intensive care situations.

\section{Methods \\ Objective: The first objective of the present study was to determine whether an NGAL immunoassay developed for a standardized clinical platform (ARCHITECT ${ }^{\ominus}$ ana- lyzer, Abbott Diagnostics) correlates with the research- based assay. The second objective was to determine the utility of the standardized NGAL immunoassay as a predictive biomarker of $\mathrm{AKI}$ after $\mathrm{CPB}$ in a large prospective cohort. \\ Design: Prospective cohort study \\ Setting: Children's hospital at a large US academic medical center. \\ Subjects: 196 children undergoing elective CPB for surgical correction or palliation of congenital heart lesions between January 2004 and June 2006. \\ Intervention: None. \\ Outcomes: The primary outcome variable was the development of AKI, defined as a $50 \%$ or greater increase}

*Corresponding author: kellumja@Upmc.edu

${ }^{3}$ Professor, Department of Critical Care Medicine, University of Pittsburgh School of Medicine, Pittsburgh, Pennsylvania, USA

Full list of author information is available at the end of the article in serum creatinine from baseline. Other outcomes included percent change in serum creatinine, days in AKI, dialysis requirement, length of hospital stay, and mortality.

\section{Results}

In a pilot study with 136 urine samples (NGAL range, 0.3 to $815 \mathrm{ng} / \mathrm{ml}$ ) and 6 calibration standards (NGAL range, 0 to $1000 \mathrm{ng} / \mathrm{ml}$ ), NGAL measurements by research ELISA and by the ARCHITECT assay were highly correlated $(r=0.99)$. In a subsequent study, serial urine NGAL measurements were obtained by ARCHITECT assay. Of the 196 children undergoing CPB, AKI developed in 99 patients (51\%), but the diagnosis using serum creatinine was delayed by 2 to $3 \mathrm{~d}$ after $\mathrm{CPB}$. In contrast, mean urine NGAL levels increased 15-fold within $2 \mathrm{~h}$ and by 25 -fold at 4 and $6 \mathrm{~h}$ after CPB. For the 2-h urine NGAL measurement, the area under the curve was 0.95, sensitivity was 0.82 , and the specificity was 0.90 for prediction of AKI using a cutoff value of $100 \mathrm{ng} / \mathrm{ml}$. The 2-h urine NGAL levels correlated with severity and duration of AKI, length of stay, dialysis requirement, and death.

\section{Conclusions}

Accurate measurements of urine NGAL are obtained using the ARCHITECT ${ }^{\odot}$ platform. Urine NGAL is an early predictive biomarker of AKI severity after CPB.

\section{Commentary}

Acute kidney injury (AKI) is estimated to occur in 5\% of hospitalized patients and as many as two thirds of intensive care unit patients [2]. Early detection of acute kidney injury could facilitate timely intervention before irreversible injury has occurred, with the goal of limiting morbidity and mortality. Unfortunately, the current diagnosis of AKI is based predominantly on changes in serum creatinine, which may not manifest for several hours after the initial insult. There is an ongoing search to identify early biomarkers of AKI that would play a role similar to that of troponin in acute myocardial infarction. Neutrophil Gelatinase-Associated Lipocalin (NGAL) is 
rapidly released by renal tubules in response to injury. Urinary NGAL is an early predictor of AKI in a variety of acute clinical settings [3]. Most of the studies evaluating NGAL as a predictor of AKI used a research-based ELISA assay. The availability of a standardized clinical platform for NGAL measurement would make this promising new biomarker more widely available.

The current study by Bennett and colleagues was conducted to accomplish two goals [1]: The first was to determine whether a new standardized clinical assay for urinary NGAL correlated closely with the research assay. The second was to determine whether urinary NGAL levels obtained using the new clinical assay could accurately predict AKI after cardiopulmonary bypass (CBP) in children. The authors successfully accomplished both goals. The new clinical assay was highly correlated with the research assay $(r=0.99)$ and highly predictive of postCPB AKI. A urinary NGAL threshold of $100 \mathrm{ng} / \mathrm{ml}$ at just two hours post-CPB predicted the subsequent development of AKI with an area under the receiver operating characteristic (ROC) curve of 0.95 and a corresponding sensitivity and specificity of 0.82 and 0.90 . Furthermore, this same 2-hour NGAL level correlated with severity and duration of AKI, length of stay, dialysis requirement, and death.

This was a well-done study showing the promise of a new clinical assay and affirming the significant delay in diagnosing AKI via changes in serum creatinine, which did not show statistical differences until 48 hours after CPB. Though this study was limited to children undergoing $\mathrm{CPB}$, subsequent studies and a meta-analysis have confirmed the utility of both urinary and plasma/serum NGAL for the early detection of AKI in other patient populations, including adults [4] and in mixed medicalsurgical intensive care unit patients [5]. Importantly, the relationship between urinary NGAL and AKI after CBP in adults varies with baseline renal function [6]. Postoperative NGAL best indentified AKI in patients with baseline glomerular filtration rates (GFR) of 90 to $120 \mathrm{ml} / \mathrm{min}$. In patients with baseline GFR $<60 \mathrm{ml} / \mathrm{min}$, urinary NGAL did not differ at any time between those who did and did not develop AKI.

\section{Recommendation}

NGAL and other novel biomarkers such as cystatin C [7] can lead to rapid detection of AKI. A strategy of early biomarker-driven detection and subsequent intervention may lead to improved outcomes and warrants further study.

\section{Competing interests}

The authors declare no competing interests

\section{Author Details}

'Clinical Fellow, Department of Critical Care Medicine, University of Pittsburgh School of Medicine, Pittsburgh, Pennsylvania, USA. ${ }^{2}$ Assistant Professor, Department of Critical Care Medicine, University of Pittsburgh School of Medicine, Pittsburgh, Pennsylvania, USA. 3Professor, Department of Critical Care Medicine, University of Pittsburgh School of Medicine, Pittsburgh, Pennsylvania, USA.

\section{Published: 23 August 2010}

\section{References}

1. Bennett M, Dent CL, Ma Q, Dastrala S, Grenier F, Workman R, Syed H, Ali S, Barasch J, Devarajan P: Urine NGAL predicts severity of acute kidney injury after cardiac surgery: a prospective study. Clin J Am Soc Nephrol 2008, 3:665-673.

2. Hoste EA, Clermont G, Kersten A, Venkataraman R, Angus DC, De Bacquer D, Kellum JA: RIFLE criteria for acute kidney injury are associated with hospital mortality in critically ill patients: a cohort analysis. Crit Care 2006, 10:R73.

3. Devarajan P: Neutrophil gelatinase-associated lipocalin--an emerging troponin for kidney injury. Nephrol Dial Transplant 2008, 23:3737-3743.

4. Haase M, Bellomo R, Devarajan P, Schlattmann P, Haase-Fielitz A: Accuracy of neutrophil gelatinase-associated lipocalin (NGAL) in diagnosis and prognosis in acute kidney injury: a systematic review and meta-analysis. Am J Kidney Dis 2009, 54:1012-1024.

5. Cruz DN, de Cal M, Garzotto F, Perazella MA, Lentini P, Corradi V, Piccinni P, Ronco C: Plasma neutrophil gelatinase-associated lipocalin is an early biomarker for acute kidney injury in an adult ICU population. Intensive Care Med 2010, 36:444-451.

6. Mcllroy DR, Wagener G, Lee HT: Neutrophil gelatinase-associated lipocalin and acute kidney injury after cardiac surgery: the effect of baseline renal function on diagnostic performance. Clin J Am Soc Nephrol 2010, 5:211-219.

7. Haase-Fielitz A, Bellomo R, Devarajan P, Story D, Matalanis G, Dragun D, Haase $M$ : Novel and conventional serum biomarkers predicting acute kidney injury in adult cardiac surgery--a prospective cohort study. Crit Care Med 2009, 37:553-560.

doi:10.1186/cc9071

Cite this article as: Gabbard W, et al.: NGAL: an emerging tool for predicting severity of AKI is easily detected by a clinical assay. Critical Care 2010, 14:318. 